\title{
Management of exfoliative glaucoma: challenges and solutions
}

Gábor Holló'

Andreas Katsanos ${ }^{2}$

Anastasios GP Konstas ${ }^{3}$

'Department of Ophthalmology, Semmelweis University, Budapest, Hungary; ${ }^{2}$ Department of

Ophthalmology, University of loannina, loannina, ${ }^{3}$ First and Third Departments of Ophthalmology, Aristotle University of Thessaloniki, Thessaloniki, Greece
Correspondence: Gábor Holló

Department of Ophthalmology, Semmelweis University, Mária u 39,

1085 Budapest, Hungary

$\mathrm{Tel}+36208258461$

Email hollo.gabor@med.semmelweisuniv.hu
This article was published in the following Dove Press journal:

Clinical Ophthalmology

22 May 2015

Number of times this article has been viewed
Abstract: Exfoliative glaucoma is the most common type of secondary open-angle glaucoma worldwide. It is characterized by high intraocular pressure (IOP) and worse 24-hour IOP characteristics. In order to minimize progression, treatment of exfoliative glaucoma has to provide a low long-term mean IOP and good 24-hour IOP control. To achieve these goals, fixed-dose combination eye drops, argon and selective laser trabeculoplasty, and various forms of surgery (trabeculectomy, deep sclerectomy, viscocanalostomy, ab interno trabeculotomy, trabecular aspiration, and cataract surgery) all need to be considered during the long-term management of the disease. Since exfoliative glaucoma is a disease of the elderly, and is frequently associated with systemic vascular disease, interdisciplinary consultations are of great clinical importance. These management aspects and the current medical, laser, and surgical results are covered in this review, with a special focus on the needs of the general ophthalmologist.

Keywords: exfoliative glaucoma, pseudoexfoliative glaucoma, medication, laser trabeculoplasty, deep sclerectomy, viscocanalostomy, trabeculectomy

\section{Introduction}

When evaluated globally, exfoliative or pseudoexfoliative glaucoma (XFG) is the most common secondary open-angle glaucoma, and is considered a disease of the elderly. ${ }^{1}$ The estimated global number of individuals with XFG varies between 5 and 6 million. XFG typically presents after 60 years of age, and in most cases significant optic nerve head and visual field damage is already present at the time of diagnosis, at least in one eye. ${ }^{2,3}$ XFG develops from exfoliation (pseudoexfoliation) syndrome (XFS), which is a genetically determined systemic condition. By definition, in XFS, both the optic nerve head and the visual field are normal (independently from the measured intraocular pressure [IOP] value). In contrast, patients with XFG demonstrate glaucomatous optic nerve head damage, in addition to elevated IOP. Amongst the main pathophysiological features of XFG are elastosis, ie, a disturbance of elastin metabolism, and abnormal synthesis and degradation of several other components of the extracellular material. ${ }^{4}$ The most characteristic clinical findings comprise the synthesis and gradual accumulation of an abnormal protein (exfoliation or pseudoexfoliation material) on the anterior lens capsule, pupil margin, zonules, and anterior chamber angle, along with liberation and deposition of pigment throughout the anterior segment of the eye. The presence of Sampaolesi's line (undulating lines of irregular pigmentation anterior to Schwalbe's line in the anterior chamber angle) is also typical in eyes with XFS or XFG. ${ }^{2}$ In some cases, exfoliation material can be clinically detectable in only one eye. However, even in apparently unilateral cases, the presence of exfoliation material can be ultrastructurally detected in both eyes as well as in other organ systems. ${ }^{2,3}$ In $38 \%$ of unilateral cases, XFS becomes clinically detectable in the clinically uninvolved 
eye within a decade. ${ }^{5}$ The details of the clinical picture of XFS and XFG have been comprehensively described in the literature. ${ }^{6-9}$ Figure 1 illustrates the typical slit-lamp appearance of XFS.

XFS represents a clinically significant risk for IOP elevation. The relative risk for clinically significant IOP elevation is 3.4-67 times higher for eyes with XFS than for eyes without XFS. 5

In contrast with XFS, XFG is not genetically determined. The known risk alleles of the single nucleotide polymorphisms of the lysyl oxidase-like protein 1 represent nearly $100 \%$ of the population at risk for XFS, but conversion from XFS to XFG is considered a result of environmental factors that increase oxidative stress in the anterior chamber. ${ }^{4,9,10}$ Elevated levels of oxidative stress markers (eg, 8-iso prostaglandin F2 $\alpha$, endothelin 1) and a decrease in antioxidant protection molecules (eg, a low ascorbic acid concentration) have been shown in the anterior segment of eyes with XFS or XFG. ${ }^{10}$ These changes are considered to be the underlying reasons for nuclear cataract formation, dysfunction of the trabecular meshwork, and in addition to elastosis, they may play a role in the development of zonular fragility, which can result in lens subluxation or luxation leading to secondary angle closure glaucoma. ${ }^{2}$ It has recently been shown that the lamina cribrosa becomes more vulnerable in XFG due to elastosis. ${ }^{4,11}$ This is of clinical significance since mean, peak, and fluctuation of 24-hour IOP in XFG are considerably higher than in primary open-angle glaucoma (POAG), and the long-term intervisit IOP variation is also greater. ${ }^{6}$ The vulnerability of the lamina cribrosa (ie, decreased resistance against mechanical deformation)

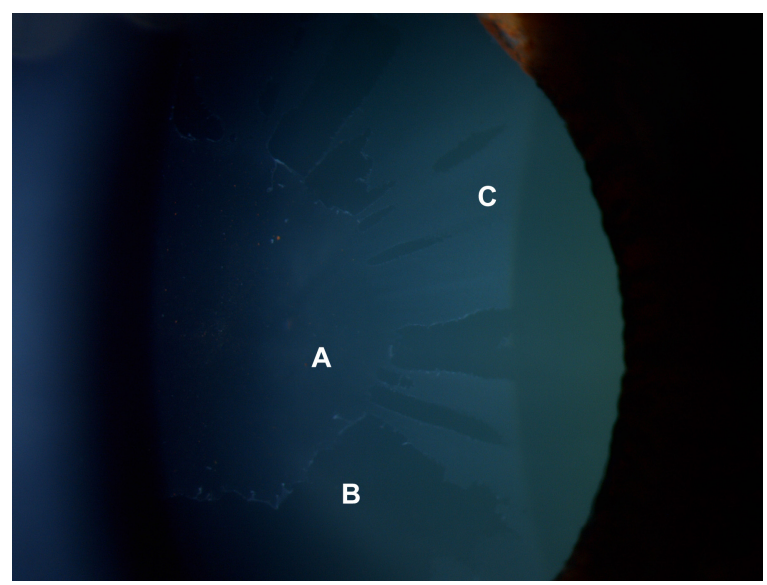

Figure I Fully developed ("classic") pattern of distribution of exfoliation material on the anterior lens capsule.

Notes: (A) Central disc of exfoliation material with pigment granules released from the pupillary ruff (B), clear intermediate zone, and $(\mathbf{C})$ a granular, layered peripheral zone. exacerbates optic nerve head damage induced by the unfavorable IOP profile. Consequently, progression in XFG without treatment is three times more rapid than that seen in untreated POAG. ${ }^{12}$ To provide long-term disease stability, a low mean IOP $(<17 \mathrm{mmHg})$ and a narrow 24-hour IOP fluctuation are needed in the long run. ${ }^{13,14}$ Since conversion from XFS to XFG occurs in approximately $30 \%$ of cases in a decade, ${ }^{5}$ careful follow-up for detection of IOP elevation and early glaucomatous structural and functional changes is mandatory in individuals with XFS.

\section{Specific aspects of IOP-lowering therapy in exfoliative glaucoma}

Currently, the only effective treatment for XFG is a substantial mean IOP reduction which also entails clinically meaningful 24-hour IOP control. ${ }^{2,6}$ Treatment of XFG has disease-specific aspects that need to be considered for successful long-term disease management. These aspects are as follows:

- A greater reduction of mean IOP combined with adequate control of 24-hour IOP characteristics (peak and fluctuation of IOP). ${ }^{2,3}$

- A greater risk of IOP elevation after pharmacological pupil dilation due to increased pigment release from the iris. ${ }^{8}$ This necessitates an additional IOP evaluation 1 or 2 hours after pupil dilation or use of preventive IOPlowering medication.

- Consideration of age-related decline in systemic tolerability of beta blockers. ${ }^{15,16}$ This is important since XFG is typically a disease of the elderly.

- Preference for fixed-dose combinations over unfixed combinations in order to reduce the number of daily instillations; this supports patient adherence to prescribed medication. ${ }^{17}$

- Consideration of dry eye syndrome and ocular surface disease, which are common in advanced age and in eyes chronically treated with preserved topical medications. ${ }^{18}$

- Value of IOP reduction achieved via removal of exfoliation material and pigment from the trabecular meshwork (phacoemulsification cataract surgery, $a b$ interno trabeculotomy, trabecular aspiration). This can be substantial, but in most cases is only temporary, since the synthesis and deposition of exfoliation material is continuous. ${ }^{2,6}$

- Gradual or sudden elevation of IOP in XFG eyes despite employing a previously successful IOP-lowering therapy, laser, or surgery, due to disease-related progression. Thus, regular follow-up is needed even in seemingly well-controlled eyes. ${ }^{2,6}$ 
- Specific need for consultation with other specialists for comanagement of XFG-related systemic vascular disease. ${ }^{19,20}$

\section{Medical therapy for exfoliative glaucoma}

Compared with POAG, achieving a sufficiently low 24-hour target IOP with medical therapy is more difficult in XFG. ${ }^{3,6,8}$ For example, with timolol monotherapy, only $8 \%$ of XFG patients were successfully controlled after 3 years of treatment, while the corresponding figure was 33\% for POAG. $^{21}$

Topical therapy with a prostaglandin analog offers the convenience of once-daily dosing (which can be easily performed by a family member when the necessary skill for self-instillation is lacking in certain elderly patients), and is free of the systemic side effects associated with topical beta blockers (eg, timolol). ${ }^{15,16}$ In a 3-month, prospective, parallelgroup, single-masked, multicenter study in XFG patients, evening-dosed latanoprost $0.005 \%$ provided somewhat greater IOP reduction and smaller diurnal IOP fluctuation than monotherapy with timolol $0.5 \%$ given twice daily. ${ }^{22}$ Although the difference in diurnal IOP between the study arms did not reach statistical significance $(17.4 \mathrm{mmHg}$ versus $18.3 \mathrm{mmHg}$ from untreated baseline values of $24.9 \mathrm{mmHg}$ and $24.7 \mathrm{mmHg}$, respectively), the IOP decrease in the morning was significantly greater, both statistically and clinically, with latanoprost therapy $(-8.5 \mathrm{mmHg}$ versus $-6.0 \mathrm{mmHg}$ ).

Optimal evaluation of the efficacy of medication is ideally based on 24-hour IOP monitoring. In a 6-month, prospective, single-masked, parallel-group study with only three time points ( $8 \mathrm{am}, 10 \mathrm{am}$, and $4 \mathrm{pm}$ ), evening-dosed latanoprost $0.005 \%$ and travoprost $0.004 \%$ were compared with the dorzolamide/timolol fixed combination given twice daily in patients with XFG. ${ }^{23}$ The mean daytime untreated IOP varied between $23.8 \mathrm{mmHg}$ and $25.7 \mathrm{mmHg}$ in all groups, and the mean IOP decrease was $8.2 \mathrm{mmHg}$ for latanoprost, $9.3 \mathrm{mmHg}$ for travoprost, and $11.5 \mathrm{mmHg}$ for the dorzolamide/ timolol fixed combination. This result, however, may not characterize the real 24-hour IOP decrease, since two of the three time points probably represented the post-instillation peak efficacy period for the dorzolamide/timolol fixed combination treatment arm.

In a complete 24-hour, prospective, single-masked, crossover study in XFG patients, travoprost $0.004 \%$ and latanoprost $0.005 \%$ reduced the untreated 24 -hour mean IOP (25.1 $\mathrm{mmHg}$ ) by $7.8 \mathrm{mmHg}(31.1 \%)$ and $7.3 \mathrm{mmHg}(30.8 \%)$, respectively. ${ }^{24}$ The between-treatment 24-hour efficacy difference was statistically significant, and was mainly due to the IOP difference in the late afternoon $(6 \mathrm{pm})$ when the mean IOP was significantly lower $(16.7 \mathrm{mmHg})$ with travoprost than with latanoprost $(17.9 \mathrm{mmHg}, P<0.01)$.

In a 3-month, prospective, single-masked, crossover, multicenter study, diurnal IOP control with bimatoprost $0.03 \%$ and latanoprost $0.005 \%$ was compared in 129 consecutive patients with XFG. ${ }^{25}$ In this study, the untreated mean diurnal IOP was $26.9 \mathrm{mmHg}$. Bimatoprost provided a clinically and statistically significant lower mean diurnal IOP than latanoprost. The mean diurnal IOP values were $17.6 \mathrm{mmHg}$ and $18.6 \mathrm{mmHg}$ for bimatoprost and latanoprost, respectively. This difference is of clinical significance since a mean diurnal IOP of $<17 \mathrm{mmHg}$, shown to be associated with the lowest rate of progression in $\mathrm{XFG},{ }^{13}$ was seen in only 34 patients (26.4\%) in the latanoprost treatment period, but was observed in 55 patients $(42.6 \%)$ in the bimatoprost treatment period.

It is noteworthy that although prostaglandin monotherapies provide greater 24-hour IOP reduction from an untreated baseline in XFG compared with $\mathrm{POAG},{ }^{26}$ a particularly low target IOP is needed to prevent progression in XFG. ${ }^{13}$ Thus, combined therapy is more often needed in XFG, and generally provides greater IOP reduction and lower mean IOP than monotherapies. Nevertheless, more controlled evidence is needed on optimal stepwise therapy for XFG in the future.

In the last decade, the role of fixed combinations in the treatment of XFG has considerably increased. In a 2-month, prospective, single-masked, crossover study, the efficacy of the dorzolamide/timolol fixed combination administered twice daily was compared with that of latanoprost $0.005 \%$ administered in the evening to treatment-naïve exfoliation patients. ${ }^{27}$ Peak IOP reduction was evaluated at $10 \mathrm{am}$, and was found to be similar with the two medications. From a mean untreated IOP value of $31.2 \mathrm{mmHg}$, dorzolamide/ timolol decreased IOP by $42.8 \%$ to $18.1 \mathrm{mmHg}$, whereas latanoprost reduced IOP by $40.2 \%$ to $18.9 \mathrm{mmHg}$. These results indicate that the 2-hour post-dose peak effect of the dorzolamide/timolol fixed combination and the 14-hour postdose effect of latanoprost monotherapy are very similar. This was the first published study to investigate the efficacy of a fixed combination as first-choice therapy in high-pressure, at-risk exfoliation patients. At the same time, the results suggest that an even greater IOP decrease can be obtained with a higher baseline pressure in XFG.

The efficacy of prostaglandin/timolol fixed combinations (latanoprost $0.005 \%$, travoprost $0.004 \%$, bimatoprost 
$0.03 \%$, or tafluprost $0.0015 \%$ combined with timolol $0.5 \%$ ) have been investigated in XFG. ${ }^{28-31}$ These fixed combinations offer meaningful IOP lowering, convenient once-daily administration, and a reduced systemic exposure to timolol compared with twice-daily administration. ${ }^{22,27}$ All these fixed combinations have demonstrated superior IOP-lowering ability compared with their constituents, and decreased exposure to preservatives when compared with use of preserved concomitant therapies. Currently, the travoprost/ timolol fixed combination is commercially available with an alternative preservative (polyquaternium 1), which eliminates the benzalkonium chloride-induced ocular surface toxicity. ${ }^{17}$ The tafluprost/timolol fixed combination is manufactured in a preservative-free unit-dose dispenser, thus eliminating all preservative-related toxicity. ${ }^{32}$ In a 3-month, 24-hour crossover study conducted specifically in XFG patients, the travoprost/timolol fixed combination showed greater 24-hour efficacy $(34.4 \%,-9.8 \mathrm{mmHg})^{28}$ than the latanoprost/timolol fixed combination $(31.2 \%,-8.9 \mathrm{mmHg})$. Both fixed combinations were administered in the evening. ${ }^{28}$

In another study, the 24-hour mean baseline IOP in a mixed XFG and POAG cohort was $27.7 \mathrm{mmHg}$. The mean 24-hour IOP reduction achieved with the travoprost/timolol fixed combination administered in the morning was $31 \%$ $(-8.6 \mathrm{mmHg})$ after 2 months of treatment. ${ }^{29}$ In a 3 -month, prospective, single-masked, multicenter, 24-hour crossover study, ${ }^{30}$ the efficacy of morning-dosed bimatoprost/timolol was compared with that of an evening-dosed bimatoprost/ timolol fixed combination in XFG. Evening dosing of the fixed combination of bimatoprost/timolol provided significantly better 24-hour IOP efficacy $(35.3 \%,-10.2 \mathrm{mmHg})$ than morning dosing $(33.9 \%,-9.8 \mathrm{mmHg})$. More recently, the IOP-lowering efficacy of the preservative-free tafluprost/ timolol fixed combination was evaluated in XFG using data for all XFG patients included in a 6-month, prospective, double-masked, active-controlled, parallel, multicenter regulatory study, in which the fixed combination was administered in the morning. ${ }^{31}$ The mean baseline IOP varied between $26.5 \mathrm{mmHg}(4 \mathrm{pm})$ and $28.1 \mathrm{mmHg}$ ( $8 \mathrm{am})$. The mean time-wise IOP decrease ranged between $8.6 \mathrm{mmHg}$ and $10.2 \mathrm{mmHg}(31.8 \%$ to $36.7 \%)$, and the overall IOP decrease was $34.1 \%$.

The above results demonstrate that an IOP reduction of $31 \%-36 \%$ from untreated baseline is achieved with the various prostaglandin/timolol fixed combinations administered once daily. Currently, no controlled data on combinations of three or more IOP-lowering products (eg, a prostaglandin/ timolol fixed combination and a topical carbonic anhydrase inhibitor, or a topical carbonic anhydrase inhibitor/timolol fixed combination and a prostaglandin analog) are available specifically in XFG. The efficacy obtained with the current monotherapies and fixed combinations in XFG is summarized in Table 1.

\section{Argon laser trabeculoplasty in exfoliative glaucoma}

Argon laser trabeculoplasty (ALT) has been used for the treatment of open-angle glaucoma since $1979 .{ }^{33}$ This was the first type of photocoagulative laser therapy of the trabecular meshwork. Other photocoagulative lasers have also been widely used for trabeculoplasty, with similar effectiveness. ${ }^{34}$ ALT exerts its IOP-lowering effect by enhancing aqueous humor outflow through the trabecular meshwork. Originally, it was hypothesized that the mechanism of action is mechanical stretching of the collapsed trabecular meshwork. ${ }^{33,34}$ However, enhancement of extracellular matrix turnover due to biological stimulation of trabecular cells by laser therapy has also been suggested. ${ }^{34}$ For successful ALT, energy absorption by melanin within the trabecular meshwork plays an important role. Since eyes with XFG typically exhibit moderate to dense pigmentation in at least part of the trabecular meshwork, ALT can be considered in most cases.

A Goldmann-type gonioscopy lens (or a specialized lens such as the Ritch trabeculoplasty lens) is used for delivery of approximately 50 evenly spaced laser burns along $180^{\circ}$ of the anterior pigmented trabecular meshwork. Typical settings with a Goldmann gonioscopy lens are a $50 \mu \mathrm{m}$ spot-size and a 0.1 second duration. The laser power should be titrated according to tissue response (varying between less than $500 \mathrm{~mW}$ for a heavily pigmented meshwork to approximately $1,000 \mathrm{~mW}$ for a lightly pigmented meshwork), so that mild blanching or very small bubble formation is observed. The efficacy of the procedure can only be assessed 4-6 weeks after treatment. Care should be taken to prevent or adequately treat the IOP spikes that frequently occur within a few hours after treatment. Oral acetazolamide, topical apraclonidine, and/or timolol have all been recommended for prevention of the IOP spikes. ${ }^{34}$ After ALT, use of topical corticosteroids (eg, dexamethasone four times daily) is recommended in addition to IOP-lowering medication for 4-7 days. ${ }^{34}$

In high-pressure XFG eyes, ALT frequently provides a large IOP reduction in the early post-laser period for months or even years. ${ }^{35-39}$ This is partly due to the high pretreatment IOP. However, the efficacy of the procedure gradually diminishes over time, possibly due to continuous deposition of exfoliation material and pigment granules within the trabecular 
Table I Intraocular pressure decrease in exfoliative glaucoma achieved with monotherapies and fixed dose combinations, based on diurnal average values

\begin{tabular}{|c|c|c|c|c|c|c|}
\hline Topical therapy & $\begin{array}{l}\text { Instillation } \\
\text { time }\end{array}$ & $\begin{array}{l}\text { Untreated baseline } \\
\text { IOP }(\mathrm{mmHg})\end{array}$ & $\begin{array}{l}\text { Mean IOP } \\
\text { decrease }(\mathrm{mmHg})\end{array}$ & $\begin{array}{l}\text { Mean IOP } \\
\text { decrease (\%) }\end{array}$ & Comment & Reference \\
\hline \multicolumn{7}{|c|}{ Prostaglandin analogs } \\
\hline Latanoprost $0.005 \%$ & Evening & 31.2 & -12.5 & -40.2 & $\begin{array}{l}\text { 14 hours after } \\
\text { instillation }\end{array}$ & 27 \\
\hline Latanoprost $0.005 \%$ & Evening & 24.9 & -7.5 & -30.1 & & 22 \\
\hline Latanoprost $0.005 \%$ & Evening & 23.8 & -8.2 & -34.4 & & 23 \\
\hline Latanoprost $0.005 \%$ & Evening & 25.1 & -7.3 & -30.8 & & 24 \\
\hline Latanoprost $0.005 \%$ & Evening & 26.9 & -8.3 & -30.8 & & 25 \\
\hline Travoprost $0.004 \%$ & Evening & 25.4 & -9.3 & -36.6 & & 23 \\
\hline Travoprost $0.004 \%$ & Evening & 25.1 & -7.8 & -31.1 & & 24 \\
\hline Bimatoprost $0.03 \%$ & Evening & 26.9 & -9.3 & -34.6 & & 25 \\
\hline \multicolumn{7}{|l|}{ Beta-blocker } \\
\hline Timolol 0.5\% & Twice daily & 24.7 & -6.4 & -25.9 & & 22 \\
\hline \multicolumn{7}{|c|}{ Prostaglandin analog/timolol fixed combination } \\
\hline Latanoprost/timolol & Evening & 28.5 & -8.9 & -31.2 & & 28 \\
\hline Travoprost/timolol & Evening & 28.5 & -9.8 & -34.4 & & 28 \\
\hline Travoprost/timolol & Morning & 27.7 & -8.6 & -31.0 & $\begin{array}{l}\text { Mixed XFG/POAG } \\
\text { population }\end{array}$ & 29 \\
\hline Bimatoprost/timolol & Morning & 29.03 & -9.84 & -33.9 & & 30 \\
\hline Bimatoprost/timolol & Evening & 29.03 & -10.2 & -35.3 & & 30 \\
\hline Tafluprost/timolol & Morning & 26.5 to 28.1 & -8.62 to -10.25 & -31.8 to -36.7 & $\begin{array}{l}\text { Mean time-wise IOP } \\
\text { and IOP decrease }\end{array}$ & 31 \\
\hline \multicolumn{7}{|c|}{ Topical carbonic anhydrase inhibitor/timolol fixed combination } \\
\hline Dorzolamide/timolol & Twice daily & 31.2 & -13.3 & -42.8 & Peak effect & 27 \\
\hline Dorzolamide/timolol & Twice daily & 25.7 & -11.5 & -44.7 & Peak effect & 23 \\
\hline
\end{tabular}

Abbreviations: IOP, intraocular pressure; POAG, primary open-angle glaucoma; XFG, exfoliative glaucoma.

meshwork, which results in a decrease of the outflow facility. In a retrospective study ${ }^{39}$ the efficacy of ALT was investigated in 28 POAG patients and 26 XFG patients who were on maximal tolerated medical treatment prior to ALT. The authors performed Kaplan-Meier survival analysis to examine the cumulative probability of failure. Failure was defined as an IOP $>22 \mathrm{mmHg}$ 4-6 months following ALT, or the need for filtering surgery. They reported that despite an initially better response to ALT, exfoliation patients eventually failed more rapidly than POAG patients. According to this study, the cumulative probability of success after approximately 8 months was $77 \%$ and $59 \%$ for the POAG and XFG patients, respectively.

In another retrospective chart review, ${ }^{36}$ the efficacy of ALT was investigated in 66 POAG eyes (mean follow-up 27 months) and 29 XFG eyes (mean follow-up 23 months). The authors established that XFG patients had a greater percent IOP reduction in the first year post-laser when compared with the POAG patients. The percent IOP decrease varied between $28 \%$ and $38 \%$ in the XFG group (mean pre-laser IOP $25.8 \mathrm{mmHg}$ ), and between $19 \%$ and $27 \%$ in the POAG group (mean pre-laser IOP $23.2 \mathrm{mmHg}$ ). The authors employed several different "failure modes" to describe the long-term efficacy of the procedure. Using "failure mode 4" (need for glaucoma-filtering surgery, a third laser treatment, IOP $\geq 22$ $\mathrm{mmHg}$, or two IOP elevations up to $15 \%$ of the baseline IOP value) the one-year failure rates for POAG and XFG were $40 \%$ and $18 \%$, respectively, while the 3 -year failure rates for the same groups were $58 \%$ and $47 \%$, respectively $(P=0.89)$. They concluded that the effectiveness of ALT decreased over time, and by 3 years, both types of glaucoma had similar success rates.

Initially, ALT was used as a treatment option in patients insufficiently controlled with maximally tolerated medical therapy. However, as clinical experience and published research demonstrated the procedure to be safe and efficacious (at least over a period of a few years), ALT has been proposed as a primary treatment in patients with open-angle glaucoma. ${ }^{40} \mathrm{~A}$ retrospective study investigated the efficacy of ALT as primary therapy in 93 POAG patients and $75 \mathrm{XFG}$ patients who were followed for a mean duration of 4.1 years. ${ }^{35}$ Based on individualized clinical criteria regarding the need for additional IOP-lowering therapy, the probability of success (defined as no need for additional therapy) was 
$80 \%$ after 2 years, $54 \%$ after 5 years, and $36 \%$ after 8 years in the XFG group. These data suggest that clinicians can expect an approximately $20 \%-40 \%$ IOP reduction from ALT during the first 4 post-laser years in XFG eyes with a pre-laser IOP of $23-30 \mathrm{mmHg} .{ }^{35-37,39}$

\section{Selective laser trabeculoplasty in exfoliative glaucoma}

Selective laser trabeculoplasty (SLT) was introduced in 1998. It utilizes a frequency-doubled Q-switched Nd-YAG laser designed to deliver relatively low energy in brief energy pulses in a large spot diameter $(400 \mu \mathrm{m})$ to the surface of the trabecular meshwork. ${ }^{41}$ According to its inventors, SLT selectively targets pigmented trabecular meshwork cells without photocoagulation. ${ }^{41,42}$ Nevertheless, the exact mechanism of SLT, by which an enhancement of aqueous humor outflow is achieved, is yet to be clarified. In contrast with ALT, SLT does not cause a thermal effect, so does not produce coagulative or disruptive changes in the trabecular meshwork. ${ }^{41}$ This is the basis for the suggestion that SLT can be successfully repeated in eyes that have previously undergone ALT or SLT. ${ }^{41,42}$ In SLT, a Goldmann-type gonioscopy lens (or a specialized trabeculoplasty lens) is used for the treatment, which usually extends to $180^{\circ}$ of the trabecular meshwork in one session. Approximately 50 laser exposures are used. ${ }^{42}$ The pulse duration is set at 3 nanoseconds, the spot-size at $400 \mu \mathrm{m}$, and the emitted energy is adjusted between 0.3 and $1.5 \mathrm{~mJ}$ to the level just below that producing bubbles. In contrast with ALT spots, the SLT spots are large and encompass the whole width of the trabecular meshwork. Tissue blanching is not observed with SLT. Since IOP spikes after SLT are not uncommon, preventive IOP-lowering treatment and topical corticosteroid or non-steroid anti-inflammatory drops are recommended for the postoperative days, similar to ALT. ${ }^{42}$

It has been shown that the efficacy of SLT is comparable with that of ALT in eyes with POAG. ${ }^{42}$ However, published information on the comparative efficacy of the two procedures in XFG is limited. A 6-month randomized trial compared the efficacy of SLT and ALT in 76 eyes of 60 patients with XFG or XFS and elevated IOP, which were all poorly controlled with topical medication. ${ }^{43}$ The baseline IOP for the SLT and ALT groups was $23.1 \mathrm{mmHg}$ and $25.2 \mathrm{mmHg}$, respectively $(P=0.03)$. The authors found that IOP reduction with SLT and ALT was similar (6.8 $\mathrm{mmHg}$ and $7.7 \mathrm{mmHg}$, respectively, $P=0.56$ ), and that a similar proportion of exfoliation patients (73\%) showed an IOP reduction of at least $20 \%$ with both treatments at the 6-month post-laser visit.
In addition, no difference between the groups was seen at the end of the follow-up period with regard to the number of IOPlowering medications. More recent studies suggest that XFG eyes respond to SLT similarly to POAG eyes, whether the procedure is used as an adjunctive $\mathrm{e}^{44,45}$ or primary ${ }^{46}$ treatment. Future research is needed to better document the efficacy of SLT specifically in XFG patients.

Despite their limited long-term efficacy, both ALT and SLT can be considered in elderly patients with suboptimal compliance; in contraindication against IOP lowering medication or poor tolerance of medical therapy; and in eyes that are poor candidates for filtration surgery. Laser trabeculoplasty does not decrease quality of life, and can be considered as primary therapy in early-to-moderate XFG. On the other hand, it is important to emphasize that laser trabeculoplasty may not be sufficient in at-risk XFG patients with advanced visual field and disc damage, who often require a low target IOP $(<17 \mathrm{mmHg})$ in the long run. ${ }^{13}$ The characteristics and results of studies using laser trabeculoplasty in XFG are summarized in Table 2.

\section{Laser iridotomy in exfoliative glaucoma}

In XFG, lens dislocation due to zonular damage may occasionally cause secondary angle closure via a pupillary block mechanism. ${ }^{7,8}$ In such cases, Nd:YAG laser iridotomy is indicated. The iridotomy technique and subsequent management do not differ from those used in primary angle closure and primary angle closure glaucoma.

\section{Surgical management of exfoliative glaucoma}

\section{Trabeculectomy}

Glaucoma surgery is typically considered when medical or laser therapy has failed to lower IOP to the desired target level; when disease progression is documented or anticipated despite maximal tolerated medical and laser therapy; when there is intolerance to medical therapy; and in cases of inadequate adherence. Trabeculectomy (typically combined with 5-fluorouracil, or more commonly with mitomycin C) has been widely considered as the gold standard surgery in advanced or progressing XFG. ${ }^{47}$ Trabeculectomy may provide the low IOP level often necessary in $\mathrm{XFG} .{ }^{48}$ On the other hand, vitreous loss due to zonular damage, a tendency for a more pronounced inflammatory reaction, hyphema from microneovascularization of the iris, synechiae formation, clinically significant choroidal detachment and choroidal hemorrhage, and cataract formation can complicate 


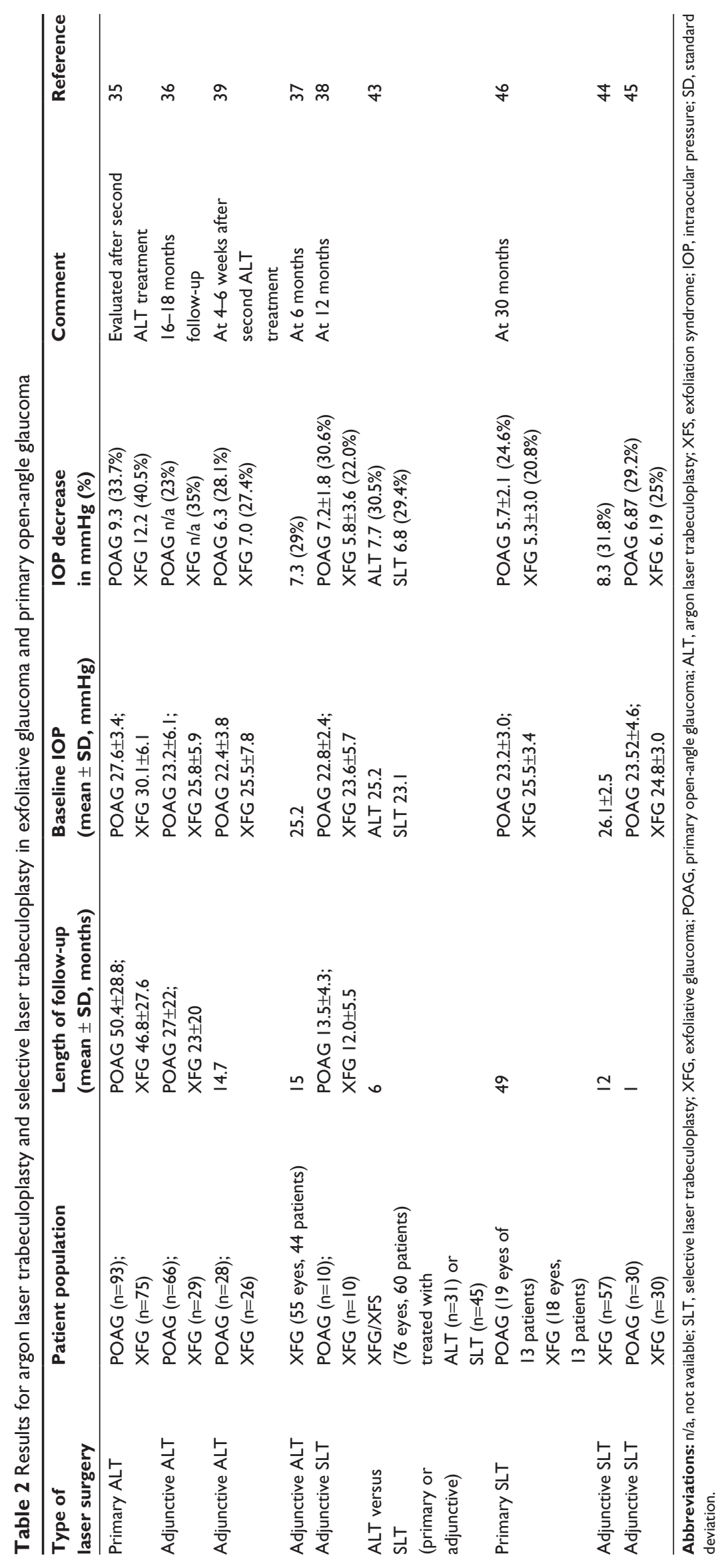


trabeculectomy in XFG. ${ }^{47}$ Nonetheless, studies have shown that the long-term success of trabeculectomy in XFG may be better than that documented with POAG. ${ }^{48-50}$ Furthermore, exfoliation patients may progress at a lower rate than POAG patients after filtering surgery. ${ }^{50}$

\section{Non-penetrating surgery}

Although trabeculectomy with adjunctive antimetabolites remains the gold standard surgical option in XFG, non-penetrating surgical techniques may also be considered in selected cases due to their favorable safety characteristics. ${ }^{51}$

\section{Deep sclerectomy}

In deep sclerectomy (DS) a "scleral lake" is created behind the Descemet's membrane underneath a partial thickness scleral flap. The aqueous humor percolates through the thin tissue of the trabeculodescemetic membrane that separates the anterior chamber from the surgically created "scleral lake", and is ultimately absorbed intrasclerally and by the subconjunctival space. To retain intrascleral space patency, use of viscoelastic material or space-maintaining implants in the "scleral lake" has been suggested. ${ }^{52}$ If aqueous humor percolation is insufficient, Nd-YAG laser goniopuncture can be performed to increase aqueous egress. The limited outflow achieved with DS ensures that hyperfiltration, hypotony, anterior chamber shallowing, cataract formation, and endophthalmitis are exceedingly rare complications. ${ }^{52}$ In a randomized study, the effectiveness of DS was increased by intraoperative application of mitomycin $\mathrm{C}$ both in POAG and XFG. ${ }^{53}$ Thirty-six months after surgery, the mean IOP in eyes operated with and without adjunctive mitomycin $\mathrm{C}$ was $15.96 \pm 1.71 \mathrm{mmHg}$ and $18.71 \pm 2.90 \mathrm{mmHg}$, respectively $(P=0.001)$. In a retrospective chart review that contained data on XFG eyes, the recently developed technique of $\mathrm{CO}_{2}$ laserassisted DS was reported to be at least as effective as conventional DS. ${ }^{54}$ The complete success rate (IOP $<18 \mathrm{mmHg}$ without medication) was $73 \%$ in the $\mathrm{CO}_{2}$ laser-assisted DS group and $71 \%$ in the control group, respectively, whereas the total success rate (IOP $<18 \mathrm{mmHg}$ with/without medication) was $96 \%$ in the $\mathrm{CO}_{2}$ laser-assisted DS group and $89 \%$ in the control group. ${ }^{54}$

No direct comparison of efficacy between trabeculectomy and DS in XFG eyes is currently available. In a prospective study of 24 XFG patients and 25 POAG patients, the efficacy of DS combined with different space-maintaining implants was evaluated. ${ }^{55}$ The mean follow-up periods were 19.9 and 16.2 months, respectively. Interestingly, DS was found to be more effective in XFG patients. The proportion of eyes with complete success (defined as IOP $<19 \mathrm{mmHg}$ without medication) was $60.7 \%$ versus $37.9 \%$ for XFG and POAG, respectively. It is noteworthy that four of the five patients with accidental intraoperative trabeculodescemetic membrane perforation had XFG. The author therefore suggested that, as with zonular and capsular fragility, eyes with exfoliation might also have a fragile trabeculodescemetic membrane.

\section{Viscocanalostomy}

This procedure is similar to DS, but involves injection of a high molecular weight viscoelastic material into Schlemm's canal. The dilation of the lumen is thought to restore aqueous humor outflow in previously non-functioning parts of Schlemm's canal and the collector channels. ${ }^{56}$ To date, there is limited information on the efficacy of viscocanalostomy specifically in XFG. In a prospective, non-randomized case series, $60 \%$ of 57 glaucomatous eyes (14 of which had XFG) had IOP $<21 \mathrm{mmHg}$ without medication after 60 months following viscocanalostomy. ${ }^{56}$ The efficacy of combined phacoemulsification and viscocanaloplasty in medically uncontrolled XFG eyes with cataract has also been investigated. ${ }^{57}$ The authors examined 30 eyes of 22 patients. The mean preoperative IOP was $25.3 \mathrm{mmHg}$ and the mean follow-up time was 18.6 months. The authors found that all eyes achieved success (defined as an IOP $<21 \mathrm{mmHg}$ with or without medication) and $90 \%$ of the eyes achieved complete success (defined as an IOP $<21 \mathrm{mmHg}$ without medication). In a prospective study, the same investigators compared the efficacy of viscocanaloplasty combined with phacoemulsification, between medically uncontrolled XFG and POAG eyes with cataract. ${ }^{58}$ The mean preoperative IOP for the POAG and XFG groups was $21.7 \mathrm{mmHg}$ and $24.5 \mathrm{mmHg}$, respectively. The mean follow-up period for both the XFG patients $(n=30)$ and the POAG patients $(n=30)$ was 19.7 months. The XFG patients had significantly lower postoperative IOP than the POAG patients $(49.7 \%$ versus $30.9 \%$ IOP reduction at the last visit, respectively).

\section{Ab interno trabeculotomy}

$\mathrm{Ab}$ interno trabeculotomy using the Trabectome ${ }^{\mathrm{TM}}$ (Neomedix Inc, Tustin, CA, USA) is a recently developed technique, and is approved for treatment of open-angle glaucoma. The instrument contains a microelectrode handpiece with irrigation and aspiration pieces. It is designed to ablate the trabecular meshwork and the inner wall of Schlemm's canal. The procedure is performed under gonioscopic view through a 1.6-1.8 $\mathrm{mm}$ size limbal side-port after the anterior chamber 
is filled with viscoelastic material. The rationale for use of this technique in XFG is that the IOP elevation is caused by accumulation of exfoliation material and pigment in the proximal part of the trabecular meshwork.

A 12-month, prospective, non-randomized trial compared the outcomes of ab interno trabeculotomy alone and in combination with cataract extraction and intraocular lens implantation, respectively, between XFG and POAG patients. ${ }^{59}$ In the ab interno trabeculotomy only analysis ( 67 cases with XFG, 450 cases with POAG), the mean preoperative IOP was $29.0 \pm 7.5 \mathrm{mmHg}$ and $25.5 \pm 7.9 \mathrm{mmHg}$, respectively $(P<0.01)$. The IOP reduction was greater in the XFG group $(12.3 \pm 8.0 \mathrm{mmHg})$ than in the POAG group $(7.5 \pm 7.4 \mathrm{mmHg}$, $P<0.01)$. The cumulative probability of success was $79.1 \%$ in XFG patients and $62.9 \%$ in POAG patients $(P=0.004)$. In the combined ab interno trabeculotomy plus cataract surgery analysis ( 45 cases with XFG, 263 cases with POAG) the mean preoperative IOP was $21.7 \pm 8.4 \mathrm{mmHg}$ and $19.9 \pm 5.4 \mathrm{mmHg}$, respectively $(P=0.06)$. The IOP decreased more in XFG patients $(7.2 \pm 7.7 \mathrm{mmHg})$ than in POAG patients $(4.1 \pm 4.6 \mathrm{mmHg}$, $P<0.01)$. Nonetheless, the cumulative probability of success was similar between the two types of glaucoma $(86.7 \%$ in $\mathrm{XFG}$ and $91.0 \%$ in POAG; $P=0.73$ ). The authors concluded that $\mathrm{ab}$ interno trabeculotomy with the Trabectome offers greater IOP reduction in XFG eyes.

\section{Trabecular aspiration}

Trabecular aspiration (TA) is a relatively new technique specifically designed for the treatment of XFG. ${ }^{60}$ In TA, aqueous humor outflow is increased via surgical removal (aspiration) of exfoliation material and pigment deposited within the outflow system. ${ }^{60}$ The specifically designed irrigation and aspiration handpieces are introduced via limbal side-ports. While the irrigation cannula ensures anterior chamber maintenance, the aspiration port is gently positioned on the trabecular meshwork. By exerting a 100-200 $\mathrm{mmHg}$ suction force for a few minutes, the extracellular debris is removed. Several clock hours of the trabecular meshwork can be treated by using a sweeping motion. The procedure is best combined with cataract surgery.

In a prospective TA study conducted in 22 eyes of 19 patients with uncontrolled $\mathrm{XFG},{ }^{60}$ the mean preoperative IOP on medication was $31.3 \mathrm{mmHg}$, which decreased significantly to $16.8 \mathrm{mmHg}$ by 18 months after TA. After surgery, $45 \%$ of the patients required no IOP-lowering medication. In the same study, the authors also evaluated 42 eyes of 36 patients with uncontrolled XFG and cataract. For these eyes, the mean preoperative IOP on medication was $32.4 \mathrm{mmHg}$, which decreased to $18.7 \mathrm{mmHg} 2$ years after combined TA and phacoemulsification or extracapsular cataract surgery. ${ }^{60}$ In this subgroup, $54 \%$ of patients were controlled without medication at the end of the 2-year follow-up period. The authors described IOP regression, which they attributed to continuous accumulation of exfoliation material and pigment within the trabecular meshwork. The complications of the procedure, such as blood reflux from Schlemm's canal and minor descemetolysis, were few and not serious. In a subsequent prospective controlled study, ${ }^{61}$ the same investigators compared the efficacy of phacoemulsification, combined phacoemulsification and TA, and combined phacoemulsification and trabeculectomy in 74 previously unoperated XFG eyes with concomitant cataract. Failure was defined as the necessity for further surgical or laser interventions (except for laser suture lysis), or need for more than one medication to achieve an individualized target pressure. After 2 years of follow-up, the proportions of successfully treated patients who underwent phacoemulsification, combined phacoemulsification and TA, or combined phacoemulsification and trabeculectomy were $36 \%, 64 \%$, and $78 \%$, respectively. The proportions of well-controlled patients who did not need any medication 2 years after the aforementioned procedures were $9 \%, 38 \%$, and $65 \%$, respectively. The results published for the various IOP-lowering surgical modalities in XFG are summarized in Table 3.

\section{Cataract surgery in exfoliative glaucoma}

Cataract surgery in eyes with exfoliation needs to be evaluated separately for two different aspects. First, cataract surgery in XFS (with or without glaucoma) is associated with a number of disease-specific surgical complications. ${ }^{62}$ These complications comprise zonular laxity and/or fragility, phacodonesis, lens subluxation or luxation, poor mydriasis, a fragile capsule, a hard nucleus, vitreous loss, and corneal endotheliopathy due to transient IOP elevation during surgery. In the postoperative period, specific complications comprise a propensity for a more pronounced inflammatory reaction (particularly after extracapsular cataract surgery), anterior capsule contraction, and delayed in-the-bag intraocular lens (subluxation or luxation). A crystalline lens or an intraocular lens luxated into the vitreous body requires pars plana vitrectomy and secondary intraocular lens implantation.

However, cataract surgery also offers a clinically significant IOP-lowering effect in patients with open-angle glaucoma, in particular in XFG and in XFS with elevated 


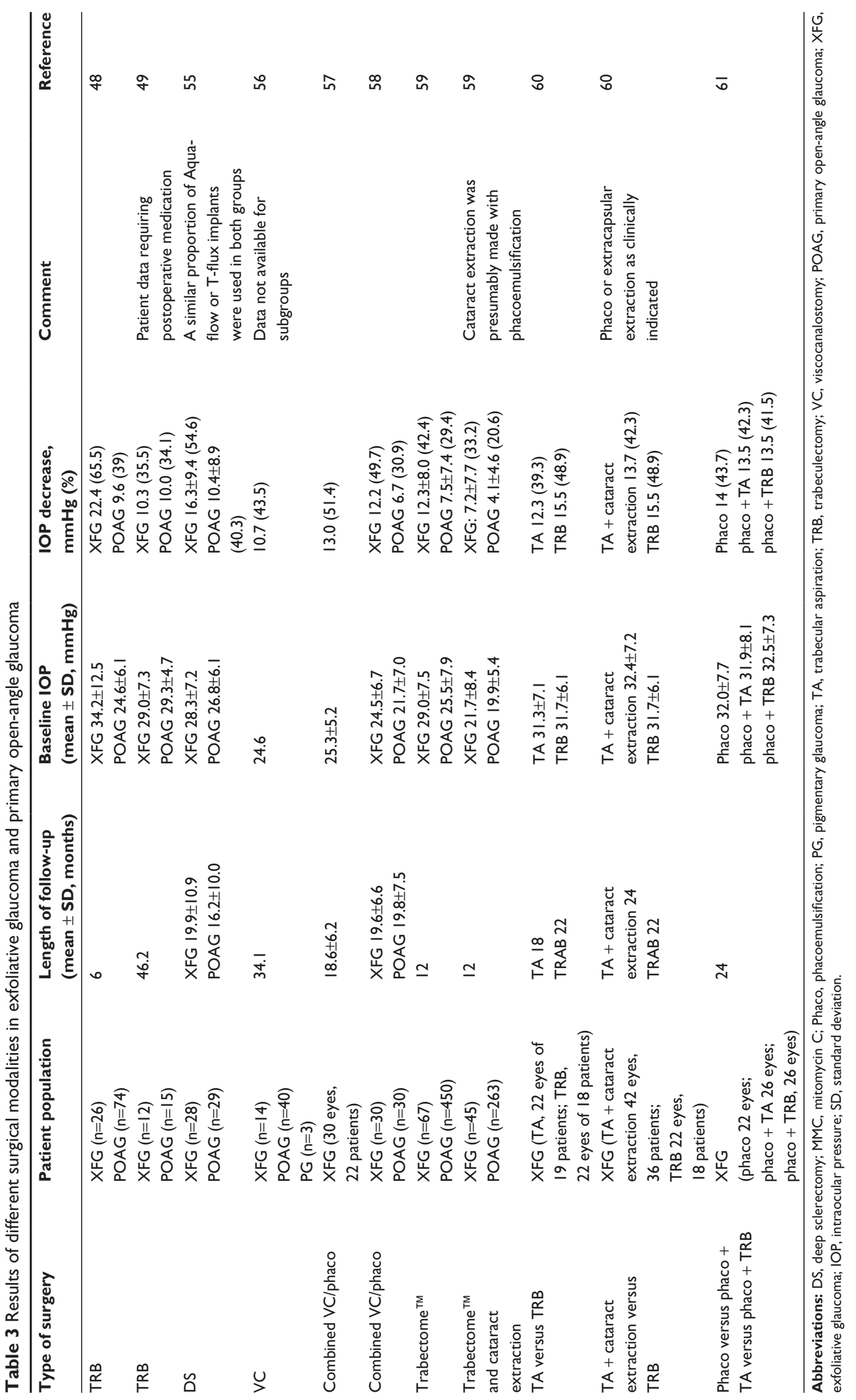


IOP ${ }^{63-66}$ It has been postulated that in these conditions the hypotensive effect of cataract surgery is due to one or more of the following changes: widening of the anterior chamber angle; a decrease in iridolenticular friction (reduced liberation of exfoliation material and pigment granules after surgery); aspiration of deposited debris from the trabecular meshwork during surgery; partial removal of the exfoliation-producing anterior capsule; and improvement in trabecular outflow as a result of low-grade inflammation (the postulated mechanism is similar to that seen after argon laser trabeculoplasty). ${ }^{62,63}$

In a 2-year, prospective, multicenter, cohort study, the ocular hypotensive effect of phacoemulsification was evaluated in 71 cataract patients with exfoliation and 112 without exfoliation. ${ }^{63}$ In the exfoliation group, 29 patients had XFG. The IOP reduction was significantly greater in the XFS and XFG patients than in both the non-glaucomatous controls and the POAG patients. Interestingly, the authors found that the ocular hypotensive effect of cataract surgery in the exfoliation group was related to the volume of irrigation fluid. This suggests that the magnitude of exfoliation material and pigment removal may be a key factor in the rate of IOP decrease after cataract surgery. The results of this prospective study corroborate the data published earlier in retrospective case series. ${ }^{64,65} \mathrm{It}$ is also noteworthy that the magnitude of postoperative IOP reduction was proportional to the preoperative IOP level in a recent retrospective study on a large cohort of 1,122 XFS and XFG patients followed for 7 years. ${ }^{66}$

\section{Need for disease-specific, targeted interventions in exfoliative glaucoma}

As discussed earlier, an increase in oxidative stress (due to an imbalance between oxidative and antioxidative factors) has been shown in the anterior segment of eyes with XFG. ${ }^{10}$ Since increased oxidative stress is probably a key factor in the development of IOP elevation in this condition, 8,10 protection against oxidative stress in the anterior segment of the eye could be a disease-specific targeted intervention. Currently, no clinically applicable specific interventions are available. Nonetheless, in one study, the aqueous humor levels of transforming growth factor- $\beta 1$ and matrix metalloproteinase- 2 were significantly lower in XFG eyes treated with latanoprost than in those treated with timolol, although the disease severity was similar in both groups. ${ }^{67}$ This finding suggests that development of topically applied antioxidant therapy may potentially be useful for a targeted XFG therapy in the future. Evaluation of all available topical prostaglandin analogs for their specific effects on biochemical processes in the anterior chamber in patients with XFG may provide a promising avenue of future research.

\section{Treatment of systemic vascular disease associated with exfoliative glaucoma}

Several clinically significant systemic and ocular vascular diseases show an association with XFS/XFG, although not in all ethnic populations. ${ }^{19,20,68,69}$ Capillary, medium and large artery dysregulation, increased plasma homocysteine levels, increased arterial stiffness and reduced distensibility, abnormal baroreflex sensitivity and heart rate regulation, increased risk for venous occlusions and aortic aneurysms have all been shown in conjunction with XFS/XFG. Management of these diseases associated with ocular exfoliation exceeds the ophthalmologist's scope. Therefore, consultation with a cardiologist, internist, or a general practitioner may be required, and could conceivably improve the standard of care for patients with exfoliation.

\section{Conclusion}

The successful long-term management of XFG remains a clinical challenge since the unique clinical attributes of this glaucoma, ie, its more rapid rate of progression and worse prognosis compared with POAG, necessitate a tailored and more proactive management strategy to prevent visual loss. Owing to a continuous synthesis of exfoliative material, a gradual buildup of pigment within the outflow system, and an extensive spectrum of pathophysiological alterations in the anterior segment of the eye (eg, increased oxidative stress, higher aqueous levels of endothelin 1), this glaucoma is characterized by worse IOP characteristics and sudden IOP rises during the course of the disease. The ophthalmologist should be aware of the need for closer monitoring of this glaucoma due to the risk of sudden loss of IOP control and the requirement for a targeted therapy. Not surprisingly, in XFG, combined therapy is needed earlier and more often. In certain at-risk cases, a fixed combination may be indicated as first-choice therapy. In the stepwise therapy paradigm for XFG, fixed-dose combinations are preferred since they enhance adherence and ocular surface health. Argon and selective laser trabeculoplasty are effective and may be selected earlier in the treatment regimen, as either primary or adjunctive therapy options. Many XFG cases, however, will require timely surgery to obtain a low target 24-hour IOP below $18 \mathrm{mmHg}$. Although trabeculectomy with an adjunctive antimetabolite remains the gold standard surgical option in XFG due to its superior efficacy, in 
the last decade other potentially safer surgical modalities have been considered in the management of XFG. There is limited controlled evidence on the long-term efficacy, safety benefits, and cost of these new surgical options. Finally, ophthalmologists need to bear in mind that XFG is also a systemic disease with a spectrum of systemic vascular sequelae. Therefore, in selected cases, consultation with the patient's cardiologist or general practitioner may be necessary.

\section{Disclosure}

The authors have no conflicts of interest in this work.

\section{References}

1. Ritch R. Exfoliation syndrome: the most common identifiable cause of open-angle glaucoma. J Glaucoma. 1994;3(2):176-178.

2. Ritch R, Schlötzer-Schrehardt U. Exfoliation syndrome. Surv Ophthalmol. 2001;45(4):265-315.

3. Konstas AGP, Holló G, Astakhov YS, et al. Presentation and longterm follow-up of exfoliation glaucoma in Greece, Spain, Russia, and Hungary. Eur J Ophthalmol. 2006;16(1):60-66.

4. Zenkel M, Krysta A, Pasutto F, Juenemann A, Kruse FE, SchlötzerSchrehardt U. Regulation of lysyl oxydase-like 1 (LOXL1) and elastinrelated genes by pathogenic factors associated with pseudoexfoliation syndrome. Invest Ophthalmol Vis Sci. 2011;52(11):8488-8495.

5. Puska PM. Unilateral exfoliation syndrome: conversion to bilateral exfoliation and to glaucoma. A prospective 10-year follow-up study. J Glaucoma. 2002;11(6):517-524.

6. Konstas AGP, Holló G, Ritch R. Exfoliative glaucoma. In: Schacknow PN, Samples RJ, editors. The Glaucoma Book. A Practical, Evidence-Based Approach to Patient Care. New York, NY, USA: Springer; 2010: 507-516.

7. Thygesen J. Ocular clinical findings in exfoliation syndrome. In: Holló G, Konstas AGP, editors. Exfoliation Syndrome and Exfoliative Glaucoma. 2nd ed. Savona, Italy: Publicomm Srl; 2012:107-114.

8. IrkecM.Clinical features of exfoliative glaucoma.In:HollóG,Konstas AGP, editors. Exfoliation Syndrome and Exfoliative Glaucoma. 2nd ed. Savona, Italy: Publicomm Srl; 2012:119-124.

9. Pasquale LR, Jiwani AZ, Zehavi-Dorin T, et al. Solar exposure and residential geographic history in relation to exfoliation syndrome in the United States and Israel. JAMA Ophthalmol. 2014;132(12):1439-1445.

10. Koliakos GG, Konstas AGP, Holló G. Biochemistry and genetics of exfoliation syndrome. In: Holló G, Konstas AGP, editors. Exfoliation Syndrome and Exfoliative Glaucoma. 2nd ed. Savona, Italy: Publicomm Srl; 2012:45-55.

11. Kim S, Sun KR, Lee JR, Lee KS. Evaluation of lamina cribrosa in pseudoexfoliation syndrome using spectral-domain optical coherence tomography enhanced depth imaging. Ophthalmology. 2013;120(9):1798-1803.

12. Heijl A, Bengtsson B, Hyman L, Leske MC; Early Manifest Glaucoma Trial Group. Natural history of open-angle glaucoma. Ophthalmology. 2009;116(12):2271-2276.

13. Konstas AGP, Holló G, Astakhov YS, et al. Factors associated with long-term progression or stability in exfoliation glaucoma. Arch Ophthalmol. 2004;122(1):29-33.

14. Quaranta L, Katsanos A, Russo A, Riva I. 24-hour intraocular pressure and ocular perfusion pressure in glaucoma. Surv Ophthalmol. 2013;58(1): 26-41.

15. Ebstein RP, Stessman J, Eliakim R, Menczel J. The effect of age on beta-adrenergic function in man: a review. Isr J Med Sci. 1985;21(3): 302-311.

16. Nieminen T, Uusitalo H, Mäenpää J, et al. Polymorphisms of genes CYP2D6, ADRB1 and GNAS1 in pharmacokinetics and systemic effects of ophthalmic timolol. A pilot study. Eur J Clin Pharmacol. 2005;61(11): 811-819.
17. Holló G, Topouzis F, Fechtner RD. Fixed-combination intraocular pressure-lowering therapy for glaucoma and ocular hypertension: advantages in clinical practice. Expert Opin Pharmacother. 2014;15(12): 1737-1747.

18. Skalicky SE, Goldberg I, McCluskey P. Ocular surface disease and quality of life in patients with glaucoma. Am J Ophthalmol. 2012;153(1):1-9.e2.

19. Holló G. Exfoliation syndrome and systemic cardiovascular diseases. J Glaucoma. 2014;23 Suppl 1:S9-S11.

20. Holló G, Konstas AGP. Exfoliation syndrome: a systemic disease. In: Holló G, Konstas AGP, editors. Exfoliation Syndrome and Exfoliative Glaucoma. 2nd ed. Savona, Italy: Publicomm Srl; 2012:87-96.

21. Blika S, Saunte E. Timolol maleate in the treatment of glaucoma simplex and glaucoma capsulare. A three-year follow up study. Acta Ophthalmol. 1982;60(6):967-976.

22. Konstas AG, Mylopoulos N, Karabatsas CH, et al. Diurnal intraocular pressure reduction with latanoprost $0.005 \%$ compared with timolol maleate $0.5 \%$ as monotherapy in subjects with exfoliation glaucoma. Eye. 2004;18(9):893-899.

23. Parmaksiz S, Yüksel N, Karabas VL, Ozkan B, Demirci G, Caglar Y. A comparison of travoprost, latanoprost, and the fixed combination of dorzolamide and timolol in patients with pseudoexfoliation glaucoma. Eur J Ophthalmol. 2006;16(1):73-80.

24. Konstas AGP, Kozobolis VP, Katsimpris IE, et al. Efficacy and safety of latanoprost versus travoprost in exfoliative glaucoma patients. Ophthalmology. 2007;114(4):653-657.

25. Konstas AGP, Holló G, Irkec M, et al. Diurnal IOP control with bimatoprost vs latanoprost in exfoliative glaucoma: a crossover observermasked 3-center study. Br J Ophthalmol. 2007;91(6):757-760.

26. Stewart WC, Konstas AGP, Nelson LA, Kruft B. Meta-analysis of 24-hour intraocular pressure studies evaluating the efficacy of glaucoma medicines. Ophthalmology. 2008;115(7):1117-1122.

27. Konstas AG, Kozobolis VP, Tersis I, Leech J, Stewart WC. The efficacy and safety of the timolol/dorzolamide fixed combination vs latanoprost in exfoliation glaucoma. Eye. 2003;17(1):41-46.

28. Konstas AGP, Mikropoulos DG, Embeslidis TA, et al. 24-hour intraocular pressure control with evening-dosed travoprost/timolol, compared with latanoprost/timolol, fixed combinations in exfoliative glaucoma. Eye. 2010;24(10):1606-1613.

29. Konstas AGP, Tsironi S, Vakalis AN, et al. Intraocular pressure control over 24 hours using travoprost and timolol fixed combination administered in the morning or evening in primary open-angle and exfoliative glaucoma. Acta Ophthalmol. 2009;87(1):71-76.

30. Konstas AGP, Holló G, Mikropoulos D, et al. Twenty-four hour intraocular pressure control with bimatoprost and the bimatoprost/timolol fixed combination administered in the morning, or evening in exfoliative glaucoma. Br J Ophthalmol. 2010;94(2): 209-213.

31. Holló G, Ropo A. Intraocular pressure decrease with preservative-free fixed and unfixed combination of tafluprost and timolol in pseudoexfoliative glaucoma. Curr Med Res Opin. 2015;31(1):13-16.

32. Holló G, Vuorinen J, Tuominen J, Huttunen T, Ropo A, Pfeiffer N. Fixed-dose combination of tafluprost and timolol in the treatment of open-angle glaucoma and ocular hypertension: comparison with other fixed-combination products. Adv Ther. 2014;31(9):932-944.

33. Wise JB, Witter SL. Argon laser therapy for open angle glaucoma. A pilot study. Arch Ophthalmol. 1979;97(2):322-399.

34. Odberg T.Laser therapy of exfoliative glaucoma. In: Holló G, Konstas AGP, editors. Exfoliation Syndrome and Exfoliative Glaucoma. 2nd ed. Savona, Italy: Publicomm Srl; 2012:151-155.

35. Odberg T, Sandvik L. The medium and long-term efficacy of primary argon laser trabeculoplasty in avoiding topical medication in open angle glaucoma. Acta Ophthalmol Scand. 1999;77(2):176-181.

36. Threlkeld AB, Hertzmark E, Sturm RT, Epstein DL, Allingham RR. Comparative study of the efficacy of argon laser trabeculoplasty for exfoliation and primary open-angle glaucoma. J Glaucoma. 1996;5(5): 311-316.

37. Sherwood MB, Svedbergh B. Argon laser trabeculoplasty in exfoliation syndrome. Br J Ophthalmol. 1985;69(12):886-890. 
38. Gracner T. Intraocular pressure response of capsular glaucoma and primary open-angle glaucoma to selective Nd:YAG laser trabeculoplasty: a prospective, comparative clinical trial. Eur J Ophthalmol. 2002;12(4): 287-292.

39. Higginbotham EJ, Richardson TM. Response of exfoliation glaucoma to laser trabeculoplasty. Br J Ophthalmol. 1986;70(11):837-839.

40. Beckman H, Meinert CL, Ritch R, Sternberg AL, Vela-Thomas Al; The Glaucoma Laser Trial Research Group. The Glaucoma Laser Trial (GLT). 2. Results of argon laser trabeculoplasty versus topical medicines. Ophthalmology. 1990;97(11):1403-1413.

41. Latina MA, Sibayan SA, Shin DH, Noecker RJ, Marcellino G. Q-switched 532-nm Nd:YAG laser trabeculoplasty (selective laser trabeculoplasty): a multi-center, pilot, clinical study. Ophthalmology. 1998; 105(11):2082-2088.

42. Barkana Y, Belkin M. Selective laser trabeculoplasty. Survey Ophthalmol. 2007;52(6):634-654.

43. Kent SS, Hutnik CM, Birt CM, et al. A randomized clinical trial of selective laser trabeculoplasty versus argon laser trabeculoplasty in patients with pseudoexfoliation. J Glaucoma. July 17, 2013. [Epub ahead of print].

44. Goldenfeld M, Geyer O, Segev E, Kaplan-Messas A, Melamed S. Selective laser trabeculoplasty in uncontrolled pseudoexfoliation glaucoma. Ophthalmic Surg Lasers Imaging. 2011;42(5):390-393.

45. Ayala M, Chen E. Comparison of selective laser trabeculoplasty (SLT) in primary open angle glaucoma and pseudoexfoliation glaucoma. Clin Ophthalmol. 2011;5:1469-1473.

46. Shazly TA, Smith J, Latina MA. Long-term safety and efficacy of selective laser trabeculoplasty as primary therapy for the treatment of pseudoexfoliation glaucoma compared with primary open-angle glaucoma. Clin Ophthalmol. 2010;5:5-10.

47. Kozobolis VP, Konstas AGP. Filtering surgery in exfoliative glaucoma. In: Holló G, Konstas AGP, editors. Exfoliation Syndrome and Exfoliative Glaucoma. 2nd ed. Savona, Italy: Publicomm Srl; 2012: 159-163.

48. Konstas AG, Jay JL, Marshall GE, Lee WR. Prevalence, diagnostic features, and response to trabeculectomy in exfoliation glaucoma. Ophthalmology. 1993;100(5):619-627.

49. Popovic V, Sjöstrand J. Course of exfoliation and simplex glaucoma after primary trabeculectomy. Br J Ophthalmol. 1999;83(3):305-310.

50. Törnqvist G, Drolsum LK. Trabeculectomies. A long-term study. Acta Ophthalmol (Copenh). 1991;69:450-454.

51. Rulli E, Biagioli E, Riva I, et al. Efficacy and safety of trabeculectomy vs nonpenetrating surgical procedures: a systematic review and metaanalysis. JAMA Ophthalmol. 2013;131(12):1573-1582.

52. Roy S, Mermoud A. Deep sclerectomy. In: Shaarawy MT, Sherwood BM, Hitchings AR, Crowston GJ, editors. Glaucoma. 2nd ed. New York, NY, USA: Elsevier-Saunders; 2015:945-959.

53. Kozobolis VP, Christodoulakis EV, Tzanakis N, Zacharopoulos I, Pallikaris IG. Primary deep sclerectomy versus primary deep sclerectomy with the use of mitomycin $\mathrm{C}$ in primary open-angle glaucoma. J Glaucoma. 2002;11(4):287-293.
54. Greifner G, Roy S, Mermoud A. Results of $\mathrm{CO}_{2}$ laser-assisted deep sclerectomy as compared with conventional deep sclerectomy. J Glaucoma. November 7, 2014. [Epub ahead of print].

55. Drolsum L. Deep sclerectomy in patients with capsular glaucoma. Acta Ophthalmol Scand. 2003;81(6):567-572.

56. Shaarawy T, Nguyen C, Schnyder C, Mermoud A. Five year results of viscocanalostomy. Br J Ophthalmol. 2003;87(4):441-445.

57. Hassan KM, Awadalla MA. Results of combined phacoemulsification and viscocanalostomy in patients with cataract and pseudoexfoliative glaucoma. Eur J Ophthalmol. 2008;18(2):212-219.

58. Awadalla MA, Hassan KM. Phacoviscocanalostomy in pseudoexfoliation glaucoma versus primary open-angle glaucoma. Can JOphthalmol. 2011;46(1):77-82.

59. Ting JL, Damji KF, Stiles MC; Trabectome Study Group. Ab interno trabeculectomy: outcomes in exfoliation versus primary open-angle glaucoma. J Cataract Refract Surg. 2012;38(2):315-323.

60. Jacobi PC, Dietlein TS, Krieglstein GK. Bimanual trabecular aspiration in pseudoexfoliation glaucoma: an alternative in nonfiltering glaucoma surgery. Ophthalmology. 1998;105(5):886-894.

61. Jacobi PC, Dietlein TS, Krieglstein GK. Comparative study of trabecular aspiration vs trabeculectomy in glaucoma triple procedure to treat pseudoexfoliation glaucoma. Arch Ophthalmol. 1999;117(10): $1311-1318$

62. Teus MA, de Benito-Llopis L. Update on cataract surgery in exfoliation syndrome. In: Holló G, Konstas AGP, editors. Exfoliation Syndrome and Exfoliative Glaucoma. 2nd ed. Savona, Italy: Publicomm Srl; 2012: 129-134.

63. Damji KF, Konstas AGP, Liebmann JM, et al. Intraocular pressure following phacoemulsification in patients with and without exfoliation syndrome: a 2-year prospective study. Br J Ophthalmol. 2006;90(8): 1014-1018.

64. Merkur A, Damji KF, Mintsioulis G, Hodge WG. Intraocular pressure decrease after phacoemulsification in patients with pseudoexfoliation syndrome. J Cataract Refract Surg. 2001;27(4):528-532.

65. Shingleton BJ, Heltzer J, O’Donoghue MW. Outcomes of phacoemulsification in patients with and without pseudoexfoliation syndrome. J Cataract Refract Surg. 2003;29(6):1080-1086.

66. Shingleton BJ, Laul A, Nagao K, et al. Effect of phacoemulsification on intraocular pressure in eyes with pseudoexfoliation: single-surgeon series. J Cataract Refract Surg. 2008;34(11):1834-1841.

67. Konstas AGP, Koliakos GG, Karabatsas CG, et al. Latanoprost therapy reduces the level of TGF beta1 and gelatinases in aqueous humour of patients with exfoliative glaucoma. Exp Eye Res. 2006;82(2): 319-322.

68. Topouzis F, Wilson MR, Harris A, et al. Risk factors for primary openangle glaucoma and pseudoexfoliative glaucoma in the Thessaloniki Eye Study. Am J Ophthalmol. 2011;152(2):219-228.

69. Andrikopoulos GK, Alexopoulos DK, Gartaganis SP. Pseudoexfoliation syndrome and cardiovascular diseases. World J Cardiol. 2014; 6(8):847-854.
Clinical Ophthalmology

\section{Publish your work in this journal}

Clinical Ophthalmology is an international, peer-reviewed journal covering all subspecialties within ophthalmology. Key topics include: Optometry; Visual science; Pharmacology and drug therapy in eye diseases; Basic Sciences; Primary and Secondary eye care; Patient Safety and Quality of Care Improvements. This journal is indexed on Submit your manuscript here: http://www.dovepress.com/clinical-ophthalmology-journa

\section{Dovepress}

PubMed Central and CAS, and is the official journal of The Society of Clinical Ophthalmology (SCO). The manuscript management system is completely online and includes a very quick and fair peer-review system, which is all easy to use. Visit http://www.dovepress.com/ testimonials.php to read real quotes from published authors. 